\title{
Oxidative Damage and Reproductive Toxicity of Organic Extracts of Jilin City Reach of the Second Songhua River on Male Rats
}

\author{
Shuguang Jin \\ School of Public Health \\ Beihua University \\ Jilin ,China \\ e-mail:bhjinshuguang12345@126.com \\ Yanli Xi \\ School of Public Health \\ Jilin Medical College \\ Jilin ,China \\ e-mail: xiyanli@126.com
}

\author{
Weiqi Sun \\ School of Public Health \\ Beihua University \\ Jilin ,China \\ e-mail: sunweiqi@126.com \\ Hongyan Wang* \\ School of Public Health \\ Beihua University \\ Jilin ,China \\ e-mail: wdj5210@163.com \\ *Corresponding author
}

\begin{abstract}
Objective: To evaluate the potential hazards of organic pollutants in Jilin City Reach of the second Songhua River. Methods: organic pollutants of water was concentrated and tested by vivo experiments on rats. Results : Testis organ coefficient decreased and sperm deformity rate increased with the increase of $\mathrm{OE}$ dose in each water samples from low water period and high water period. A significant decrease of SOD activity and a increase of MDA content of testis was observed at $50 \mathrm{~mL} / \mathrm{kg}$ group of midstream, $25 \mathrm{~mL} / \mathrm{kg}, 50 \mathrm{~mL} / \mathrm{kg}$ group of downstream from high water period. Furthermore, reproductive toxicity of organic extracts in high water period was higher that in low water period in Jilin City Reach of the second Songhua River. Conclusion : Organic pollutants of water in Jilin City Reach of the second Songhua River have potential reproductive toxicity effects.
\end{abstract}

Keywords-water pollution; organic extracts; reproductive toxicity;SOD;MDA

\section{INTRODUCTION}

Water pollution is one of the major environmental problems, organic pollutants in water is not only one of the water pollution problem to be greatly concerned for people, but also the focus of domestic and foreign scholars research in recent years. A large number of industrial waste discharged over standards, sewage directly discharged without treatment, chemical fertilizers, pesticides and other chemicals was irrational used in vast rural area, which made our rivers and water contaminated to varying degrees, resulted in deterioration of water environment quality, but also a great threat to the people's health. Although the level of Toxic organic pollutants in water was usually trace, long-term contact will cause serious health hazards to people because of long-term and cumulative effect .
The Second Songhua River flows through the city of Jilin, which is the largest tributary of the Songhua River. It is the most important water resources for Jilin Province. There was close relationship between water quality and the health of the population. To evaluate the potential hazards of organic pollutants in Jilin City Reach of the second Songhua River, organic pollutants of water was concentrated and tested by vivo experiments on rats in this study. Then Oxidative Damage of rat testis and sperm deformity test to investigate reproductive toxicity was observed to investigate the influence of reproductive toxicity of organic extracts of Jilin City Reach of the second Songhua River on male rats.

\section{MATERIALS AND METHODS}

\section{A. Reagents.}

XAD-2 resin column, dimethyl sulfoxide (DMSO) cyclophosphamide (CP) was purchased from American sigma company; dichloromethane, methanol, acetone was purchased from Tianjin reagent factory .SOD and MDA test kit were purchased from Nanjing Jiancheng Biological Engineering Institute..

\section{B. Sample collection and extraction organic compounds}

200 L water samples were respectively collected from upstream, midstream and downstream of Jilin City Reach of the second Songhua River in March (low water period) and August (high water period) in 2012.Sediment was removed through static precipitation $24 \mathrm{~h}$ In the laboratory. Organics in water was adsorbed in XAD-2 resin column cleaned with acetone. Column flow rate is about $30 \mathrm{ml} / \mathrm{min}$. The adsorption of organic compounds on the resin was eluted with $100 \mathrm{ml}$ redistilled acetone two times in 6 fractions. Eluent was put in K-D concentrator at $45^{\circ} \mathrm{C}$ 
water bath, the residue was dissolved in DMSO and volumed to $5 \mathrm{ml}$. That was to say organic extracts contained in $1 \mathrm{~mL}$ DMSO solution is equivalent to those in $40 \mathrm{~L}$ raw water samples. The organic extracts dissolved DMSO was stored at $4^{\circ} \mathrm{C}$ for further studies .

\section{Animal groups and treatment}

Male SD rats were provided by the Jilin University laboratory and were randomly divided into 11 groups with six animals in each after adaptive feeding for 1 weeks. The groups were respectively negative control(NC) group $(0.25 \mathrm{ml} / \mathrm{kg}$ DMSO), positive control(PC) group $(50 \mathrm{mg} / \mathrm{kg}$ cyclophosphamide), organic extracts exposure group $(12.5 \mathrm{~mL} / \mathrm{kg}, 25 \mathrm{~mL} / \mathrm{kg}$ and $50 \mathrm{~mL} / \mathrm{kg}$ respectively from upstream, midstream and downstream) .Rats were injected peritoneally with $0.1 \mathrm{~mL} / 20 \mathrm{~g}$ of body weight, once a day for five days.All rats were sacrificed by cervical dislocation after the last exposure 6h. bilateral testicular was separated and weighed.The organ coefficient was calculated

\section{Sperm deformity test}

The production of sperm smear and staining according to the conventional method ${ }^{[1]}$. The right epididymis was put in the dish containing $5 \mathrm{ml}$ PBS and cut into pieces standing for $5 \mathrm{Min}$, draw the sperm suspension, drops on clean slides, even push plate, air drying, ethanol fixation, $2 \%$ eosin staining $1 \mathrm{~h}$ and microscopic examination.Chubby head, shaped banana, no hook, double head, twin tail and amorphous sperm was counted in 1000 sperm each rat, and calculate the total abnormal rate

\section{E. Detection of SOD activity and MDA content in testicular tissue}

Unilateral testicular was put on the ice, film was stripped, tissue homogenate was preparated according to the ratio of quality to volume 1:9 and physiological saline into $10 \%$ homogenate. Then homogenate were high-speed centrifuged $(10000 \mathrm{r} / \mathrm{min})$ for 30 minutes at $4^{\circ} \mathrm{C}$. The homogenate supernatant was taken out. SOD activity was determined by adjacent benzene three phenol oxidation colorimetric determination, content of MDA was determined by thiobarbituric acid test kit, protein content was determined by the biuret method. The MDA content is represented by $\mathrm{nmol} / \mathrm{mgPro}$ SOD activity results indicated by $\mathrm{U} / \mathrm{mgPro}$.

\section{F. Statistical methods}

Statistical analysis was performed using SPSS 13 statistical software, Groups were compared using univariate analysis of variance.

\section{RESULTS}

\section{A. General observation}

All rats of solvent control group, positive control group and the OE group grew well and no symptoms of systemic poisoning was seen. Figure 1, 2 respectively shows the testis organ coefficient of rats effected by the organic extracts in low water period or high water period .It can be observed that compared with the negative control group, the testis organ coefficient decreased with the increasing $\mathrm{OE}$ dose in water samples from upstream, midstream and downstream. There was significant among the OE water samples from downstream in high water period with control group $(\mathrm{P}<0.05)$.
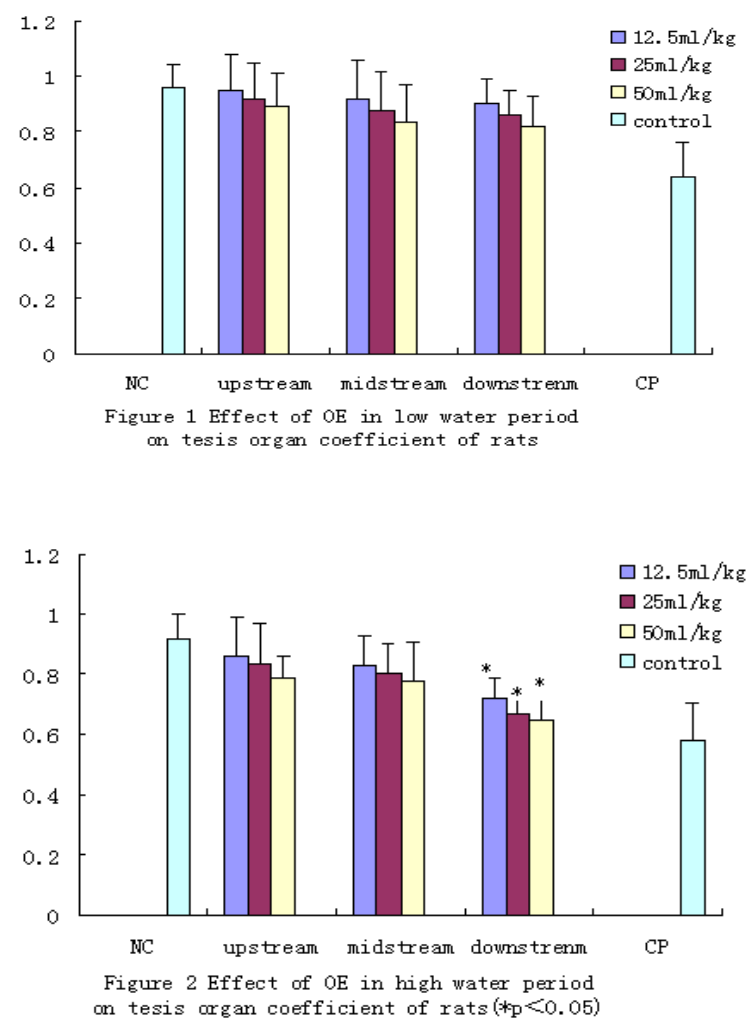

\section{B. Sperm malformation test}

It can be seen from table 1 that sperm deformity rate increased with the increase of $\mathrm{OE}$ dose in each water samples from low water period and high water period. There was no significant differences $(\mathrm{P}>0.05)$ about sperm deformity rate induced by $\mathrm{OE}$ come from low water period and upstream water of high water period compared with the control group. While sperm deformity rate induced by $\mathrm{OE}$ come from midstream and downstream water of high water period was obviously higher than the control group $(\mathrm{P}<0.05)$. The sperm deformity rate of $\mathrm{CP}$ positive control group rats was far higher than that of all the OE exposed group and the control group $(\mathrm{P}<0.05)$. 


\begin{tabular}{|c|c|c|c|}
\hline Groups & Dose & Low water period & High water period \\
\hline DMSO & & $4.24 \pm 0.94$ & $4.67 \pm 1.30$ \\
\hline \multirow[t]{3}{*}{ upstream } & $12.5 \mathrm{~m} 1 / \mathrm{kg}$ & $4.78 \pm 1.67$ & $5.25 \pm 1.67$ \\
\hline & $25 \mathrm{~m} 1 / \mathrm{kg}$ & $4.63 \pm 1.59$ & $5.98 \pm 2.14$ \\
\hline & $50 \mathrm{~m} 1 / \mathrm{kg}$ & $4.98 \pm 2.10$ & $6.32 \pm 2.08$ \\
\hline \multirow[t]{3}{*}{ midstream } & $12.5 \mathrm{~m} 1 / \mathrm{kg}$ & $5.26 \pm 2.23$ & $8.34 \pm 3.21 *$ \\
\hline & $25 \mathrm{~m} 1 / \mathrm{kg}$ & $5.78 \pm 1.98$ & $8.96 \pm 2.19 *$ \\
\hline & $50 \mathrm{~m} 1 / \mathrm{kg}$ & $6.12 \pm 2.35$ & $9.12 \pm 3.01 *$ \\
\hline \multirow[t]{3}{*}{ downstream } & $12.5 \mathrm{~m} 1 / \mathrm{kg}$ & $6.56 \pm 1.89$ & $10.78 \pm 3.57^{*}$ \\
\hline & $25 \mathrm{~m} 1 / \mathrm{kg}$ & $6.89 \pm 1.76$ & $12.65 \pm 3.54^{*}$ \\
\hline & $50 \mathrm{~m} 1 / \mathrm{kg}$ & $7.01 \pm 2.68$ & $14.89 \pm 4.05^{*}$ \\
\hline $\mathrm{CP}$ & & $24.35 \pm 3.65 \#$ & $26.87 \pm 5.12 \#$ \\
\hline
\end{tabular}

\section{MDA content of testis}

Figure 5, 6 respectively shows MDA content of rats testis effected by the organic extracts in low water period or high water period.There was no significant difference between the OE groups in low water period and the control group differences $(P>0.05)$.Compared with the negative control group, A significant increase of MDA content of testis was seen at $50 \mathrm{~mL} / \mathrm{kg}$ group from high water period and $20 \mathrm{~mL} / \mathrm{kg}$ group of midstream and downstream from high water period $(\mathrm{P}<0.05)$.

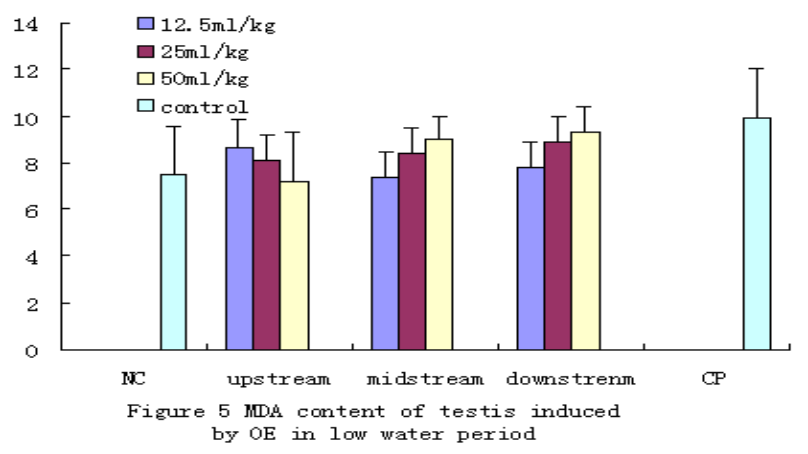

* compared with the negative control group, $\mathrm{P}<0.05$; \# compared with the other group, $\mathrm{P}<0.05$

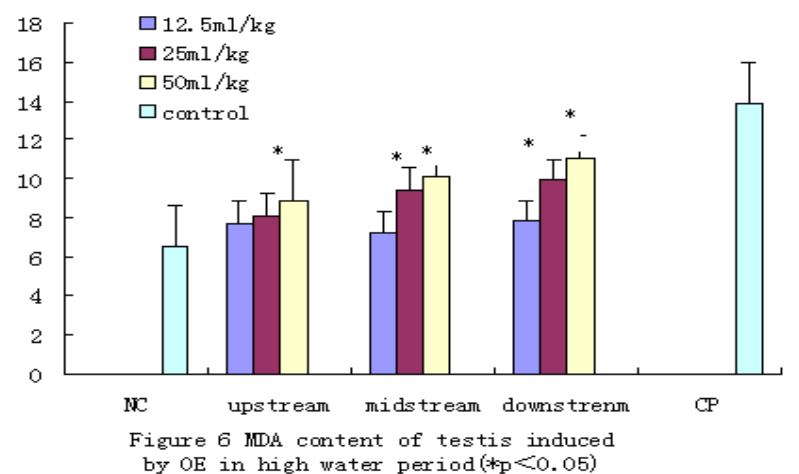

\section{DISCUSSION}

The reproductive system is sensitive to the role of foreign compounds. Reproductive system may be seen some damage before toxicity have not appeared in other system. In recent years, many studies have found that the male reproductive system is the target organ of toxicity of organic pollutants in water important ${ }^{[2]}$. In this study, organic extracts of water samples from Jilin City Reach of the second Songhua River exposed in male rats. A series of damage in reproductive system was observed in the OE dose group rats. Testis organ coefficient decreased with the increasing OE dose, especially a significant decrease of organ coefficient of testis was observed at downstream water samples from high water period .Sperm deformity rate increased with the increase of $\mathrm{OE}$ dose in each water samples from low water period and high water period. Sperm deformity rate induced by $\mathrm{OE}$ come from midstream and downstream water of high water period was obviously higher than the control group. This may be related to the estrogenic effect of organic pollutants which can interfere with gonadal hormone ${ }^{[3]}$. Compared to the negative control group, sperm deformity rate induced by $\mathrm{OE}$ come from midstream and downstream water of high water period was increased significantly, more than 2 times than the solvent control group, according to $\mathrm{Li}$ 
Shouqi ${ }^{[4]}$ s standards, we could think the dose of organic extracts of water come from midstream and downstream in high water period of Jilin City Reach of the second Songhua River has reached the the judgment standard of mouse sperm malformation mutagens. We also found that the damage in reproductive system induced by OE in high water period was significantly heavier than that in low water period at the corresponding section, the reason may be concentration of PAHs in high water period was significantly higher than that in low water period of Jilin City Reach of the second Songhua River ${ }^{[5]}$.The lower total PAHs concentration was mainly because that Jilin city is in North China, river water temperature is lower in winter, the solubility of PAHs is smaller, the ground in the winter freeze, the PAHs concentration run off into the river through surface obviously decrease.

Under normal circumstances, the oxidant and antioxidant system of the body maintain the dynamic balance, so that the active oxygen in cells maintained at lower level ${ }^{[6]}$. When Exogenous chemical substance and its metabolites poison the body, they can directly inhibit or depletion the antioxidant system of the body and lead to oxidative stress. In the toxic mechanism of organic pollutants, free radicals play an important role ${ }^{[7]}$. Superoxide dismutase (SOD) is a main component of intracellular anti free radical protection system, can remove superoxide anion, the protect the structure, function of biological macromolecules and cell membrane. The activity of SOD indirectly reflects the ability of the organism to scavenge oxygen free radicals. Malondialdehyde (MDA) is one if the end product of peroxidation, its content reflects the degree of lipid peroxidation ${ }^{[8]}$. In this study, a significant decrease of SOD activity and a increase of MDA content of testis was observed at $50 \mathrm{~mL} / \mathrm{kg}$ group of midstream, $25 \mathrm{~mL} / \mathrm{kg}$, $50 \mathrm{~mL} / \mathrm{kg}$ group of downstream from high water period. Compared with the control group, the difference was significant $(\mathrm{P}<0.05)$, Combined with the water of $\mathrm{OE}$ can lead to increase in the rate of sperm deformity. These suggested that in the high water period, the high concentration of OE produced a large amount of oxygen free radical in the testis and depleted SOD reserve of testis, free radical coming from organic metabolism may directly damage protein and genetic material ${ }^{[9]}$. This study also found that the activity of SOD of testis tissue in rats of low dose OE group come from the upstream water appeared the increased phenomenon, which suggested the organic extract of low dose could prompt Stimulation and induce tissue SOD activity increased, which belongs to the early stress change to toxicants ${ }^{[10]}$.
From this study, reproductive toxicity of organic extracts in high water period was higher that in low water period in Jilin City Reach of the second Songhua River, especially $\mathrm{OE}$ in midstream and downstream water of high water period can cause a certain degree of effect on the reproductive system of male rats. The results made us understand that organic pollutants of water in Jilin City Reach of the second Songhua River have potential reproductive toxicity effects. Due to the actual contact way of people is long-term and low dose exposure, the damage characteristics of water $\mathrm{OE}$ on the people need further confirm according to the chronic toxicity test and population epidemiology investigation

\section{ACKNOWLEDGMENT}

This work was financially supported by the Science and Technological Project of Jilin Province in China (No.201205038), the Nature and Science fund from Jilin Province Ministry of Education(No.2011129)

\section{REFERENCES}

[1] Xinru Wang . Methods of technology of toxicological test.2rd ed, Beijing: People's Medical Publishing House, 2003,pp109-129

[2] Huaijun Tian, Desheng Wu and Rui Tao,"Study of organic extract of running water on sperm testosterone content on mice," Journal of environment and health, vol.16,Feb.1999,pp.74-75

[3] Gray T J Band Beamand J A,"Effect of some phthalate esters and other testicular toxins on primary cultures of testicular cells," Fd Chem Toxicol,vol.22,Jan.1984,pp. 1231- 1232

[4] Shouqi Li. Principles and methods of toxicology . Chengdu: Sichuan University press, 2003.pp.35-42

[5] Shuguang Jin Huan Li and Jing Zhang,"Water Environmental Human Health Risk Assessment of Polycyclic Aromatic Hydrocarbons in the Jilin City Reach of the second Songhua River," Chinese rural health service management,vol.43,Oct.2014,pp.1249-1252

[6] KediYang, environmental health/environmental hygiene, 7th ed, Beijing: People's Medical Publishing House,2013,pp.103-108

[7] Muhai Nie , Quanxin Zhang and Wei Zhu ,"Effect on lipid peroxidation of rat of extracts from drinking water," Modern preventive medicine, vol.35,Aug.2008,pp.1551-1555

[8] Pant N, Pant A B and Shukla M,"Environmental and Experimental Exposure of Phthalate Esters: The Toxicological Consequence on Human Sperm," Hum \& Exp Toxicol,vol.30,Jun.2011,pp.507-514

[9] Dadoune,J P,“Expression of mamma liansper matozoal nucleoproteins," Microsc Res Tech, vol.61,Jan.2003,pp.56- 75

[10] Bo Gi and Shujin Zhao,"The general domestic research situation of the relationship between SOD detection and disease," Guangdong pharmacy, vol.12 ,Jan.2001,pp. 7-8,2003 